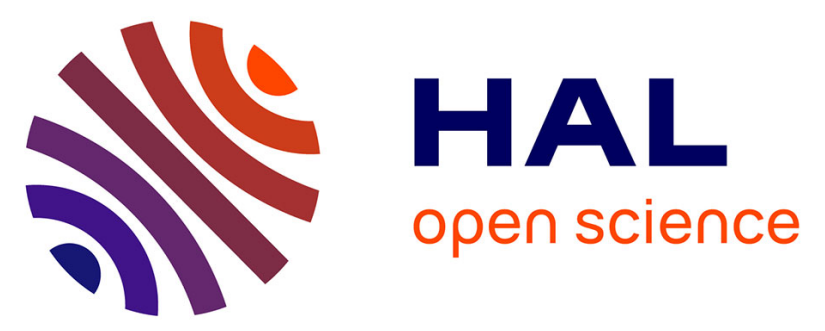

\title{
A blob-based tomographic reconstruction of 3D coronary trees from rotational x-ray angiography
}

Jian Zhou, Alexandre Bousse, Guanyu Yang, Jean-Jacques Bellanger, Limin M. Luo, Christine Toumoulin, Jean-Louis Coatrieux

\section{- To cite this version:}

Jian Zhou, Alexandre Bousse, Guanyu Yang, Jean-Jacques Bellanger, Limin M. Luo, et al.. A blobbased tomographic reconstruction of 3D coronary trees from rotational x-ray angiography. Medical Imaging 2008: Physics of Medical Imaging, Feb 2008, San Diego, CA, United States. pp.69132N-12, 10.1117/12.769478 . inserm-00335244

\section{HAL Id: inserm-00335244 https://www.hal.inserm.fr/inserm-00335244}

Submitted on 28 Oct 2008

HAL is a multi-disciplinary open access archive for the deposit and dissemination of scientific research documents, whether they are published or not. The documents may come from teaching and research institutions in France or abroad, or from public or private research centers.
L'archive ouverte pluridisciplinaire HAL, est destinée au dépôt et à la diffusion de documents scientifiques de niveau recherche, publiés ou non, émanant des établissements d'enseignement et de recherche français ou étrangers, des laboratoires publics ou privés. 


\title{
A blob-based tomographic reconstruction of 3D coronary trees from rotational X-ray angiography
}

\author{
Jian Zhou ${ }^{a, b, c, d}$, Alexandre Bousse ${ }^{a, b, c, d}$, Guanyu Yang ${ }^{a, b, c, d}$, Jean-Jacques Bellanger ${ }^{b, c, d}$, \\ Limin Luo ${ }^{a, d}$, Christine Toumoulin ${ }^{b, c, d}$ and Jean-Louis Coatrieux ${ }^{b, c, d}$ \\ ${ }^{a}$ Laboratory of Image Science and Technology (LIST), Southeast University, Nanjing, China; \\ ${ }^{b}$ INSERM, U642, Rennes, France; \\ ${ }^{c}$ Université de Rennes 1, LTSI, Rennes, France; \\ ${ }^{d}$ Centre de Recherche en Information Biomédicale Sino-francais (CRIBs), Rennes, France.
}

\begin{abstract}
A method is proposed for a 3D reconstruction of coronary networks from rotational projections that departs from motion-compensated approaches. It deals with multiple views extracted from a time-stamped image sequence through ECG gating. This statistics-based vessel reconstruction method relies on a new imaging model by considering both the effect of background tissues and the image representation using spherically-symmetric basis functions, also called 'blobs'. These blobs have a closed analytical expression for the X-ray transform, which makes easier to compute a cone-beam projection than a voxel-based description. A Bayesian maximum a posteriori (MAP) estimation is used with a Poisson distributed projection data instead of the Gaussian approximation often used in tomography reconstruction. A heavy-tailed distribution is proposed as image prior to take into account the sparse nature of the object of interest. The optimization is performed by an expectationmaximization like (EM) block iterative algorithm which offers a fast convergence and a sound introduction of the non-negativity constraint for vessel attenuation coefficients. Simulations are performed using a model of coronary tree extracted from multidetector CT scanner and a performance study is conducted. They point out that, even with severe angular undersampling (6 projections over 110 degrees for instance) and without introducing a prior model of the object, significant results can be achieved.
\end{abstract}

Keywords: cone-beam, coronary, reconstruction, X-ray, angiography, blob, maximum a posteriori, expectationmaximization

\section{INTRODUCTION}

Coronary arterial disease is a major cause of mortality especially in Europe and the US. Quantitative and accurate characterization of stenoses (length, cross-sectional area) as well as their location within the whole coronary network is thus of major importance for diagnosis and treatment. The 3D reconstruction of arterial trees enhanced with contrast agent has attracted much attention for years using $2 \mathrm{D}$ angiographic image sequences with fairly high time resolution. This problem has been addressed first through computer vision approaches using mono- and bi-plane systems with epipolar techniques and feature matching in order to find corresponding primitives and retrieve their 3D geometry. However, the resolution of this ill-posed problem requires either a prior model, ${ }^{1}$ additional views, ${ }^{2}$ or the joint use of motion. ${ }^{3}$ All the reported methods rely on a robust and accurate detection capable to provide in a first step the vessel centerlines, which is difficult to obtain without a significant user interaction (refer to ${ }^{4}$ for a review). The availability of X-ray C-arm imaging systems opens now new perspectives for this reconstruction. While the clinician already gets more insights into the full 3D anatomy during the rotation of the C-arm (covering up to 180-240 degrees within a 7-8 seconds), the full 3D reconstruction may take benefit of a higher number of projections (150-200) by using tomographic methods. Several options can be considered. One first consists to perform a motion-compensated tomographic reconstruction, inspired from Bonnet's work, ${ }^{5}$ using the motion of the coronary arteries previously estimated by means of computer vision methods. ${ }^{6}$ Such method is highly dependent on a critical pre-processing step and on the reliability of the motion estimate. Another way is to carry out the reconstruction from very few views (typically 5-8), corresponding to a single cardiac phase, selected by ECG-gating. A 3D reconstruction of centerlines has been proposed by exploring the 3D voxelized space, region growing and reprojection. ${ }^{7}$ A fully iterative method has been reported 
in ${ }^{8,9}$ by minimizing the L1-norm of the reconstructed image and a regularization based on vesselness and Gibbs smoothing priors.

This contribution describes an iterative reconstruction using a block sequential regularized EM (expectationmaximization) algorithm (BSREM $)^{10}$ for maximizing a regularized Poisson likelihood estimation, where the update is multiplicative. Instead of using a voxel basis, we consider a spherically-symmetric basis function (i.e the blob basis), the Kaiser-Bessel (KB), which has shown attractive properties. ${ }^{11}$ A 3D realistic phantom data set has been built using a coronary tree extracted from MDCT volume image. Cone beam projections have been computed from this phantom, with and without a background added coming from real angiographic projections. A performance study has then been conducted in terms of convergence, reconstruction error.

\section{METHOD}

The imaging geometry used in most recent rotational X-ray angiography systems is a cone-beam geometry. We consider a monoenergetic X-ray source, the ideal projection function due to this point source is:

$$
Y(\boldsymbol{u} ; \theta(t)) \propto \exp \left\{-\int \underline{\mu}\left(\mathcal{T}(\theta(t))\left[\boldsymbol{x}_{0}+s \cdot \frac{\boldsymbol{x}_{d}(\boldsymbol{u})-\boldsymbol{x}_{0}}{\left\|\boldsymbol{x}_{d}(\boldsymbol{u})-\boldsymbol{x}_{0}\right\|}\right] ; t\right) d s\right\}
$$

where $\boldsymbol{u}=\left[u_{1}, u_{2}\right]^{\prime} \in \mathbb{R}^{2}, \boldsymbol{x}_{0}=[0,-d, 0]^{\prime}$ and $\boldsymbol{x}_{d}(\boldsymbol{u})=\left[u_{1}, D-d, u_{2}\right]^{\prime}$ (where $d$ is the distance from the source to the center of rotation, and $D$ the source-to-detector distance), $\|\cdot\|$ is the standard Euclidean norm. $\mathcal{T}(\theta(t))$ is the three-dimensional rotation transform:

$$
\mathcal{T}(\theta(t))=\left[\begin{array}{ccc}
\cos (\theta(t)) & -\sin (\theta(t)) & 0 \\
\sin (\theta(t)) & \cos (\theta(t)) & 0 \\
0 & 0 & 1
\end{array}\right]
$$

where $\theta(t)$ gives the projection angle at time $t$. The term $\mu(\boldsymbol{x} ; t)\left(\boldsymbol{x} \in \mathbb{R}^{3}\right)$ represents the total linear X-ray attenuation coefficient corresponding to the sum of the contribution of the dyed blood vessels of interest $\mu$ and the background tissue $\mu_{\mathrm{b}}$, i.e.,

$$
\underline{\mu}(\boldsymbol{x} ; t)=\mu(\boldsymbol{x} ; t)+\mu_{\mathrm{b}}(\boldsymbol{x} ; t) .
$$

In real cases, data acquisition is usually done with several discrete time instances, e.g., $\left\{t_{1}, \ldots, t_{K}\right\}$. By letting $\theta_{k} \equiv \theta\left(t_{k}\right)(k=1, \ldots, K)$, we have a projection sequence $\left\{Y\left(\boldsymbol{u} ; \theta_{k}\right)\right\}$. From equation (3), we see that both arteries and background tissues are projected. The contribution of background tissues is significant in angiogram, and cannot be ignored. One conceptually simple way is to acquire additional projection images of background tissues, denoted by $\left\{Y_{\mathrm{b}}\left(\boldsymbol{u} ; \theta_{k}\right)\right\}$, and to perform logarithmic subtractions: $\log Y_{\mathrm{b}}\left(\boldsymbol{u} ; \theta_{k}\right)-\log Y\left(\boldsymbol{u} ; \theta_{k}\right)$. However, this is unrealistic since, at least, it requires that the $\mathrm{X}$-ray system has a very accurate repositioning so that it can provide two spatially matched sequences. In addition, any patient motion between the acquisitions will produce artifacts due to incomplete subtraction. An alternative solution is to numerically approximate the background and then subtract it.

In this paper, we adopt this latter strategy with a slightly different implementation. Suppose we already have virtual background images $\hat{Y}_{\mathrm{b}}\left(\boldsymbol{u} ; \theta_{k}\right)$. Instead of a logarithmic subtraction as shown previously, we propose the following approximation for the $k$ th projection image:

$$
Y\left(\boldsymbol{u} ; \theta_{k}\right) \propto \hat{Y}_{\mathrm{b}}\left(\boldsymbol{u} ; \theta_{k}\right) \exp \left\{-\int \mu\left(\mathcal{T}\left(\theta_{k}\right)\left[\boldsymbol{x}_{0}+s \cdot \frac{\boldsymbol{x}_{d}(\boldsymbol{u})-\boldsymbol{x}_{0}}{\left\|\boldsymbol{x}_{d}(\boldsymbol{u})-\boldsymbol{x}_{0}\right\|}\right] ; t_{k}\right) d s\right\} .
$$

The challenge still remains when performing a direct reconstruction using $\left\{Y\left(\boldsymbol{u} ; \theta_{k}\right)\right\}$. The vessel attenuation coefficient function $\mu$ is a function of time, depending on, e.g., the distribution of contrast agent, and much more important, the motion of vessels during the acquisition procedure. These effects lead to the inconsistency in projection data. We will consider in this paper that the attenuation within the vessel is constant over time and that all other motions (due to patient movement or respiration) can be neglected. Since the cardiac motion is relatively regular and periodical, through ECG gating, we are able to choose several projections from a rotational 
sequence which correspond to the same 3D heart motion. We have limited our reconstruction study to a single instant within a cardiac phase. We assume $Y\left(\boldsymbol{u} ; \underline{\theta_{k}}\right)$ 's consist of $\underline{K}$ selected projection images. Further, we can use $\mu(\boldsymbol{x})$ instead of $\mu\left(\boldsymbol{x} ; t_{k}\right)$ for conciseness. Now the task turns to how these selected angiographic images $Y\left(\boldsymbol{u} ; \underline{\theta_{k}}\right)$ 's can be utilized to reconstruct $\mu(\boldsymbol{x})$ of interest.

As stated, this problem is close to the conventional static tomography reconstruction with, however, critical features: 1) Since a usual examination of coronary angiography is often taken in a relatively short time period (e.g., typically no more than four or five cardiac cycles), the number of consistent projections is small (i.e., $\underline{K}$ is small, usually less than 6), and projections may not be equally spaced; 2) For relatively low X-ray exposition, the angular scanning is severely limited (e.g., for a typical C-arm rotational X-ray angiography system, the dynamic angle range is less than $180^{\circ}$ ). This is sharply in contrast to the conventional cone-beam tomography in which a normal scanning covers a full range of $360^{\circ}$. Therefore, a direct 3D reconstruction of vessel from rotational $\mathrm{X}$-ray angiography is indeed a very ill-posed problem. In the following section, we will discuss our approximation solution.

\subsection{System Model}

We transform the above problem into the discrete domain. A single projection image can be discretized and stored lexicographically in one vector. All projection vectors can be stacked one by one, leading to a large data vector $\boldsymbol{Y}=\left[Y_{1}, \ldots, Y_{N}\right]^{\prime}$ (where $N$ is the total number of projection data, and the prime represents the vector or matrix transpose). Only noisy data can be obtained and we assume that these data are independent random variables whose ensemble means, according to (4), can be expressed as

$$
\bar{Y}_{i}(\boldsymbol{u}) \propto Y_{\mathrm{b} i} \exp \left\{-[\boldsymbol{A u}]_{i}\right\}
$$

for all $i=1, \ldots, N$, where $Y_{\mathrm{b} i}$ is the $i$ th element of the background image vector $\boldsymbol{Y}_{\mathrm{b}}, \boldsymbol{u}=\left[\mu_{1}, \ldots, \mu_{J}\right]^{\prime}$ (where $J$ is the length of $\boldsymbol{u})$ is a discrete representation of vessel attenuation coefficient function $\mu(\boldsymbol{x}), \boldsymbol{A}$ is the cone-beam projection operator, and $[\cdot]_{i}$ returns the $i$ th entry of a vector in brackets.

Without loss of generality, we study here the vessel reconstruction within a cubic field of view. A typical image representation is to consider the piecewise continuous attenuation function $\mu(\boldsymbol{x})$ defined over the cubic region. It can be written as the superposition of scaled and shifted copies of the basis function $\Psi(\boldsymbol{x})$, as follows:

$$
\mu(\boldsymbol{x})=\sum_{j=1}^{J} \mu_{j} \Psi\left(\boldsymbol{x}-\boldsymbol{x}_{j}\right)
$$

where $\left\{\boldsymbol{x}_{j}\right\}(j=1, \ldots, J)$ form a set of spatial basis locations (or grid points as well). There are many choices of basis functions, one of which is: $\Psi(\boldsymbol{x})=1$, if $\|\boldsymbol{x}\|_{\infty} \leq 1 / 2$ else $\Psi(\boldsymbol{x})=0$ (assuming that the grid spacing is unitless 1). This is the so-called voxel basis that has a constant density inside the voxels. From a signal processing standpoint, it only provides, however, a poor interpolation. Instead of using voxel basis, we consider in this paper another basis family: the spherically-symmetric basis function (also known as the blob basis) which has the following general form:

$$
\Psi(\boldsymbol{x})=\mathcal{B}(\|\boldsymbol{x}\|)
$$

where $\mathcal{B}(\cdot)$ is a circularly symmetric kernel. The concept of blob in tomography has been applied for image reconstruction, e.g. ${ }^{12-14}$ etc. It is also widely used in the area of volume visualization, e.g., volume rendering. ${ }^{15}$ An important blob basis is known as the Kaiser-Bessel (KB) kernel, which has shown certain attractive properties. ${ }^{11}$ It can be written as

$$
\mathcal{B}_{m, \alpha, R}(r)=\left\{\begin{array}{cl}
\frac{1}{I_{m}(\alpha)}\left(\sqrt{1-(r / R)^{2}}\right)^{m} I_{m}\left(\alpha \sqrt{1-(r / R)^{2}}\right) & |r| \leqslant R \\
0 & \text { otherwise }
\end{array}\right.
$$

where $I_{m}(\cdot)$ denotes the modified Bessel function of the first kind of order $m, R$ is the radius of the blob, $\alpha$ is a non-negative real number controlling the shape of the blob. Usually, we take $m>0$ so that the blob has $m-1$ continuous derivatives at the boundary. 
A blob-based forward projection operator $\boldsymbol{A}$ can be determined according to the basic definition of X-ray projection (4). For one element $a_{i j}$, using the X-ray transform of the blob function at location $\boldsymbol{x}_{j}$ along the $i$ th ray path $\Gamma_{i}$, we have

$$
a_{i j}=\int_{\Gamma_{i}} \mathcal{B}_{m, \alpha, R}\left(\left\|\boldsymbol{x}-\boldsymbol{x}_{j}\right\|\right) d \ell
$$

Since the X-ray transform of a KB kernel leads to another KB like kernel, i.e.,

$$
\underline{\mathcal{B}}_{m, \alpha, R}(r)=R \sqrt{\frac{2 \pi}{\alpha}} \frac{I_{m+1 / 2}(\alpha)}{I_{m}(\alpha)} \mathcal{B}_{m+1 / 2, \alpha, R}(r), \quad|r| \leq R,
$$

it follows that

$$
a_{i j}=\underline{\mathcal{B}}_{m, \alpha, R}\left(\operatorname{dist}\left[\boldsymbol{x}_{j}, \Gamma_{i}\right]\right)
$$

with $\operatorname{dist}\left[\boldsymbol{x}_{j}, \Gamma_{i}\right]$ the distance between the grid point $j$ and the ray $\Gamma_{i}$.

\subsection{Image MAP estimation}

The commonly used maximum likelihood (ML) estimation finds an image reconstruction that maximizes the probability distribution of the projections, as well as the likelihood distribution. The likelihood distribution, denoted by $\log \operatorname{Pr}(\boldsymbol{Y} \mid \boldsymbol{u})$, is characterized by the imaging model. Since the nature of X-ray observations is a counting process, this paper considers a Poisson likelihood

$$
\log \operatorname{Pr}(\boldsymbol{Y} \mid \boldsymbol{u}) \propto \sum_{i}\left\{-\bar{Y}_{i}(\boldsymbol{u})+Y_{i} \log \left(\bar{Y}_{i}(\boldsymbol{u})\right)\right\}
$$

where terms independent of $\boldsymbol{u}$ has been ignored. The choice of this model is also due to another interpretation of the log-Poisson likelihood term which is the so-called Kullback-Leiber (KL) distance. The KL distance provides a measure of dissimilarity between two vectors $\boldsymbol{p}$ and $\boldsymbol{q}$, is defined by

$$
\mathrm{KL}(\boldsymbol{p}, \boldsymbol{q})=\sum_{i}\left\{p_{i} \log \left(p_{i} / q_{i}\right)-p_{i}+q_{i}\right\} .
$$

Now letting $p_{i}=Y_{i}$ and $q_{i}=\bar{Y}_{i}(\boldsymbol{u})$ and also ignoring terms independent of $\boldsymbol{u}$, it can be shown that $\log \operatorname{Pr}(\boldsymbol{Y} \mid \boldsymbol{u}) \equiv$ $-\mathrm{KL}(\boldsymbol{Y}, \overline{\boldsymbol{Y}}(\boldsymbol{u}))$ where $\overline{\boldsymbol{Y}}(\boldsymbol{u})=\left[\bar{Y}_{1}(\boldsymbol{u}), \ldots, \bar{Y}_{N}(\boldsymbol{u})\right]^{\prime}$. The proposed Poisson model is not specific: even for nonPoisson data, we are still able to seek a ML solution for which the likelihood term can be represented by $\operatorname{Pr}(\boldsymbol{Y} \mid \boldsymbol{u}) \propto \exp \{-\mathrm{KL}(\boldsymbol{Y}, \overline{\boldsymbol{Y}}(\boldsymbol{u}))\}$.

However, due to the typical limits in fidelity of data, ML estimates are often unstable, and have to be improved. Usually a unique and stable estimate is sought by incorporating prior information on the original image $\boldsymbol{u}$, leading to the well known Bayesian MAP estimation. In the MAP estimation, we are interested in the maximum of the posteriori distribution $\operatorname{Pr}(\boldsymbol{u} \mid \boldsymbol{Y})$. Using Bayes's rule, we rewrite $\operatorname{Pr}(\boldsymbol{u} \mid \boldsymbol{Y})$ as $\operatorname{Pr}(\boldsymbol{u} \mid \boldsymbol{Y}) \propto \operatorname{Pr}(\boldsymbol{Y} \mid \boldsymbol{u}) \operatorname{Pr}(\boldsymbol{u})$ where $\operatorname{Pr}(\boldsymbol{u})$ represents the image prior distribution. Then, the MAP estimation is found as

$$
\hat{\boldsymbol{u}}_{\mathrm{MAP}}=\arg \max _{\boldsymbol{u}}\{\log \operatorname{Pr}(\boldsymbol{Y} \mid \boldsymbol{u})+\log \operatorname{Pr}(\boldsymbol{u})\} .
$$

The image prior is certainly the key to any MAP estimation. It can not only provide a stable solution but also improve the quality of reconstruction, e.g., by suppressing artifacts due to limited angle projections. One prior defined over a discrete grid system relates to the discrete Markov random field (MRF) on which a roughness measure can be constructed from local neighborhoods. Typically, $\operatorname{Pr}(\boldsymbol{u})$ has the following generic form

$$
\operatorname{Pr}(\boldsymbol{u}) \propto \exp \{-\beta U(\boldsymbol{u})\}
$$

where $U(\boldsymbol{u})$ is the energy function, and $\beta$ an adjustable parameter. Let $\mathcal{N}_{j}$ be the neighborhoods of the $j$ th grid. The energy function can be written as

$$
U(\boldsymbol{u})=\sum_{j} \sum_{s \in \mathcal{N}_{j}} \omega_{j s} \phi\left(\mu_{j}-\mu_{s}\right)
$$




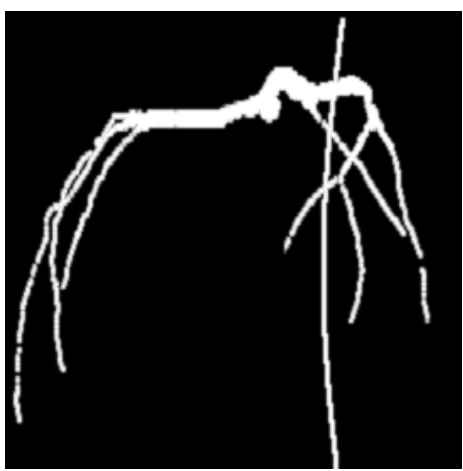

(a)

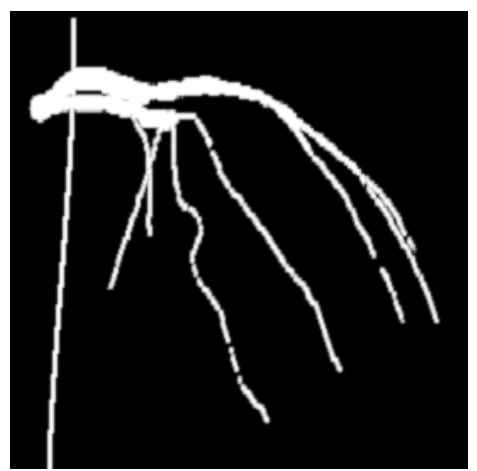

(b)

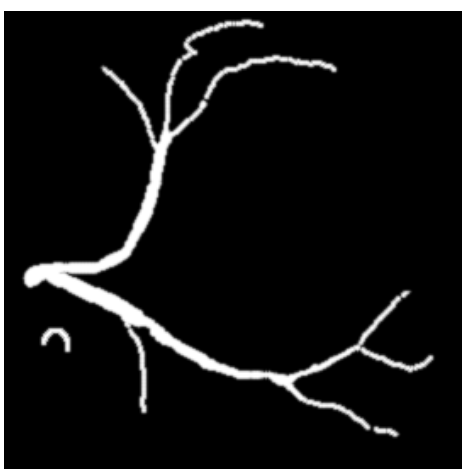

(c)

Figure 1. Computer simulated phantom. Figures from left to right are maximum intensity projections along three axes, $\mathrm{x}, \mathrm{y}, \mathrm{z}$ respectively.

where $\omega_{j s}$ is the weight for the pair of grid points $j$ and $s$. $\phi$ is the potential function, measuring the interaction between the two grid points. In coronary angiography, vessels having branch-like structures occupies a very small part of the volume, so a sparse object prior is of relevance. Now, consider the distribution of local neighborhood differences, i.e., $\mu_{j}-\mu_{s}$ : it should be sharply peaked around zero, due to the contribution of most smooth areas, and have broad tails representing the contribution of the vessels (in particular their edges). Consequently, a sparse object prior can be modeled by a heavy-tailed distribution. With this in mind, we selected a potential function $\phi(t)=|t|$. The corresponding penalty relates to the Laplacian distribution that belongs to the family of heavy-tailed distribution. Another advantage is that the resulting $\mathcal{R}(\boldsymbol{\mu})$ is close to the total variational (TV) regularization that is powerful for noise smoothing while edge preserving.

\section{SIMULATION STUDY}

\subsection{Materials}

The experiments have been conducted using phantom data. The reference left coronary arteries were extracted from a volume of cardiac MSCT reconstructions. ${ }^{16}$ Fig. 1 shows three maximum-intensity projections of the phantom along the three axes. Stenotic areas and a simulated catheter were also added.

The imaging protocol used in this paper was adapted from the C-arm rotational X-ray coronary angiography Siemens AXIOM-Artis system. The detector plane, $140^{2} \mathrm{~mm}^{2}$, which was sampled uniformly into $512^{2}$ pixels. All reconstructions have been performed in a volume of $(110 \mathrm{~mm})^{3}$. This field of view was also uniformly sampled into $128^{3}$ grid points that served as blob locations. According to our discussion on the choice of blob basis, we have used $m=2, \alpha=6.0$, and $R=1.5$ blob spacing for the calculation of operator $\boldsymbol{A}$. Six cone-beam vessel projection images, without background tissues, uniformly spaced over the range $120^{\circ}$ were first generated (the starting angle is $-29.0^{\circ}$ ). Then, we used the method of low order polynomials approximation to create the required background images $\hat{\boldsymbol{Y}}_{\mathrm{b}}$ (called later on the "true backgound") from some real angiography images acquired separately on the Siemens device. The mean observation data were produced according to the model (4). Poisson noisy data were then generated, which were used for reconstructions. Fig. 2 shows some examples of noisy projection images at different angular views.

\subsection{Reconstruction}

We focus our study on the iterative reconstruction algorithm. The image reconstruction was performed by BSREM. ${ }^{10}$ In this algorithm, a fully iteration is divided into a set of subiterations, each being performed sequentially using one of the predetermined blocks of measurement data. In our simulation study, the data within each projection image were grouped into one block. Therefore, this leads to a total number of six blocks. The BSREM algorithm is an extension of RAMLA (row-action maximum likelihood algorithm) ${ }^{17}$ for maximizing a regularized Poisson likelihood estimation as (14). They relate to the well-known algebraic reconstruction technique (ART), ${ }^{18}$ but the update is multiplicative (rather than additive) and a grouped projection can be performed simultaneously. BSREM has been shown even faster with guaranteed global convergence properties. ${ }^{10}$ 

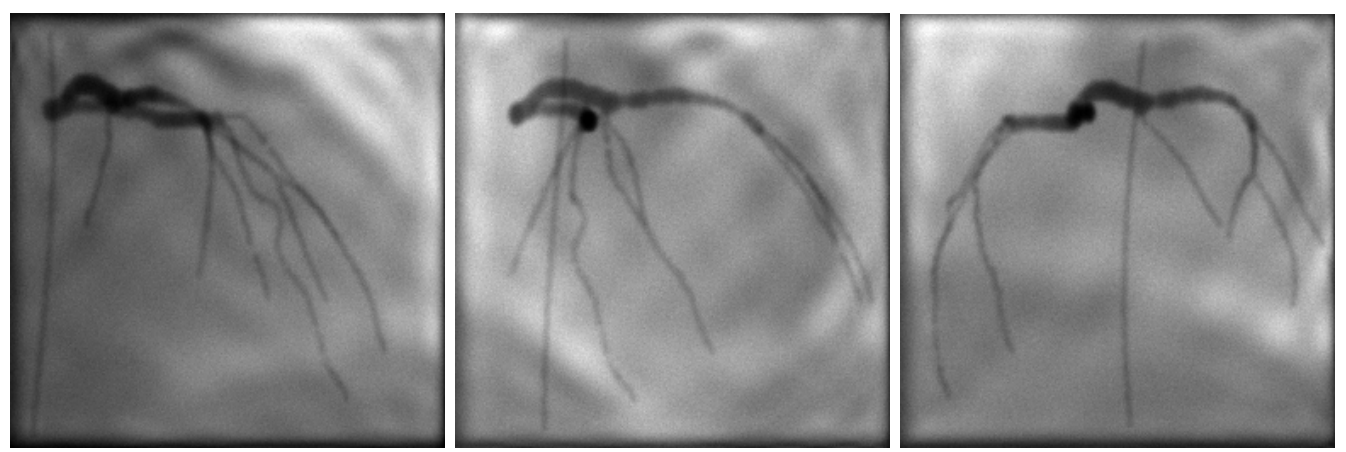

Figure 2. Simulated noisy projection images at angle: (a) $-29.0^{\circ}$, (b) $18.2^{\circ}$, and (c) $65.4^{\circ}$, respectively.

\subsection{Influence of background images}

As discussed in Section 2, one can construct a set of new measurement data by using logarithm substraction. Let $\tilde{\boldsymbol{Y}}=-\log \left(\boldsymbol{Y} / \hat{\boldsymbol{Y}}_{\mathrm{b}}\right)$. Then, $\tilde{\boldsymbol{Y}}$ serves as the approximate observations of projections $\boldsymbol{A u}$. $\operatorname{In}^{6}{ }^{8}$ the authors have used $\tilde{\boldsymbol{Y}}$ to reconstruct the vessels of interest. This method was evaluated in this paper and compared with our strategy as expressed in (4). While the resulting data $\tilde{\boldsymbol{Y}}$ cannot be Poisson distributed, we still can use the BSREM algorithm but the data fidelity term should be interpreted as the KL distance, yielding a modified BSREM for image reconstruction from the presubtracted measurement data. We named it here the PreSub.+BSREM algorithm.

For comparison, we introduced two measures of error: 1) the global mean square error defined by

$$
\operatorname{MSE}_{\text {global }}(n)=\frac{\left\|\hat{\boldsymbol{u}}^{n}-\boldsymbol{u}^{\mathrm{ref}}\right\|^{2}}{\left\|\boldsymbol{u}^{\mathrm{ref}}\right\|^{2}} \times 100 \%
$$

which provides the normalized percentage error between the $n$th iteration $\hat{\boldsymbol{u}}^{n}$ and the reference volume $\boldsymbol{u}^{\text {ref }}$. 2 ) the vessel mean square error denoted by $\operatorname{MSE}_{\text {vessel }}(n)$ defined in a similar way except that the error is only measured within the support of vessels instead of the entire field of view.

Tab. 1 shows the global MSE values of the two different algorithms at several selected iteration number. We see that the smaller $\mathrm{MSE}_{\text {global }}$ can be obtained by the proposed BSREM. This possibly means that the logarithm substraction operation imposed before data processing may destroy the optimality of statistical based iterative algorithm, leading to a suboptimal one in terms of global mean square error. Fig. 3 shows the evolution of $\mathrm{MSE}_{\text {vessel }}$ during the iteration progress. Again, BSREM without pre-logarithm substraction shows a slight better performance.

As mentioned already, the background images are usually unavailable in practice, and they can only be estimated from the measured data. To obtain virtual projection images of background tissues, we have adapted the method used in. ${ }^{6,8}$ First, we created the binary vessel mask. This is can be done by thresholding. Second, a morphological closure operation was applied to the noisy projection images to remove vessels. Here, the structure element was a disk whose radius has been chosen large enough to cover the blood vessels. Then, a virtual background image is obtained by combining the original projection image and the filtered image: the virtual background image takes the pixel value from the filtered image if the pixel belongs to a vessel according to the binary vessel mask images, otherwise we use the value on the original projection image. Fig. 4 shows some results when creating one virtual background image from the projection image at angle $-5.4^{\circ}$.

The estimated background images, denoted by $\overline{\hat{\boldsymbol{Y}}}_{\mathrm{b}}$, were combined with either BSREM or PreSub.+BSREM to investigate the effect of background tissues on reconstructions. Fig. 5 compares the maximum intensity

Table 1. Comparison of $\mathrm{MSE}_{\text {global }}$ values (\%) for PreSub+BSREM and BSREM (both with $\beta=0$ ) at several iterations.

\begin{tabular}{c|c|c|c|c}
\hline \#Itr. & 5 & 30 & 70 & 100 \\
\hline PreSub+BSREM & 36.7 & 22.3 & 19.3 & 18.5 \\
BSREM & 52.1 & 18.7 & 16.6 & 16.5 \\
\hline
\end{tabular}




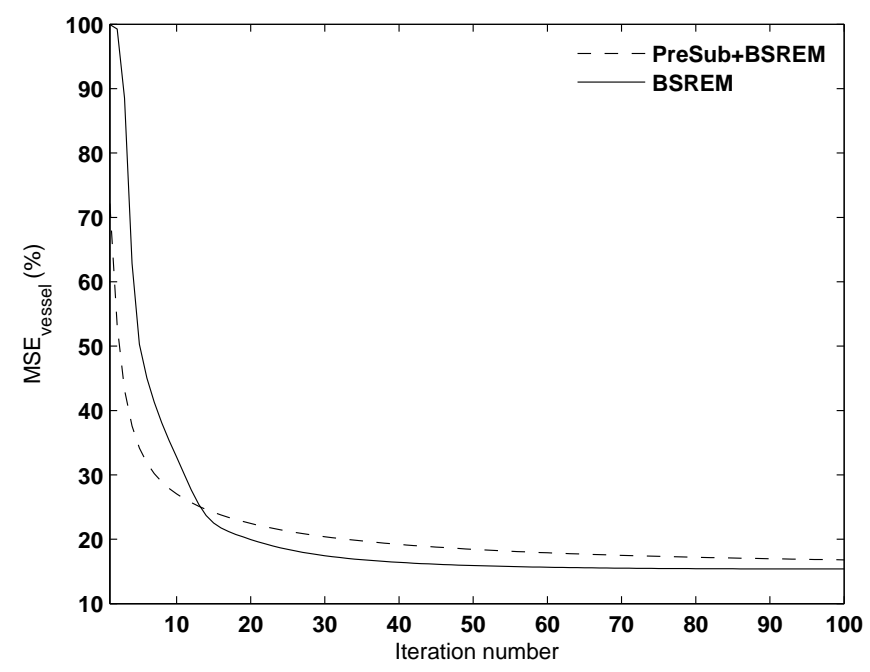

Figure 3. A comparison of $\mathrm{MSE}_{\text {vessel }}$ for PreSub+BSREM and BSREM ( $\beta=0$ for both algorithms) as the function of iteration. Here uses true background images $\hat{\boldsymbol{Y}}_{\mathrm{b}}$.

projection images with either true or virtual background images. Noise and artifacts are clearly shown and this confirms that the accuracy of background images does affect the reconstruction quality. Nevertheless, they are mainly found in background regions, and most of the vascular structures can be preserved. Tab. 2 lists some $\mathrm{MSE}_{\text {vessel }}$ values yielded by the two approaches. The results seem to justify the same fact that the proposed BSREM can behave better than the one with pre-logarithm subtraction. However, it can be observed that the mean square error becomes larger over iterations. This is a common feature of iterative ML estimation that can produce an overfit to noisy data, leading to degeneration of reconstruction. An introduction of regularization (or setting $\beta>0$ as well) should improve the solution and will be discussed next.
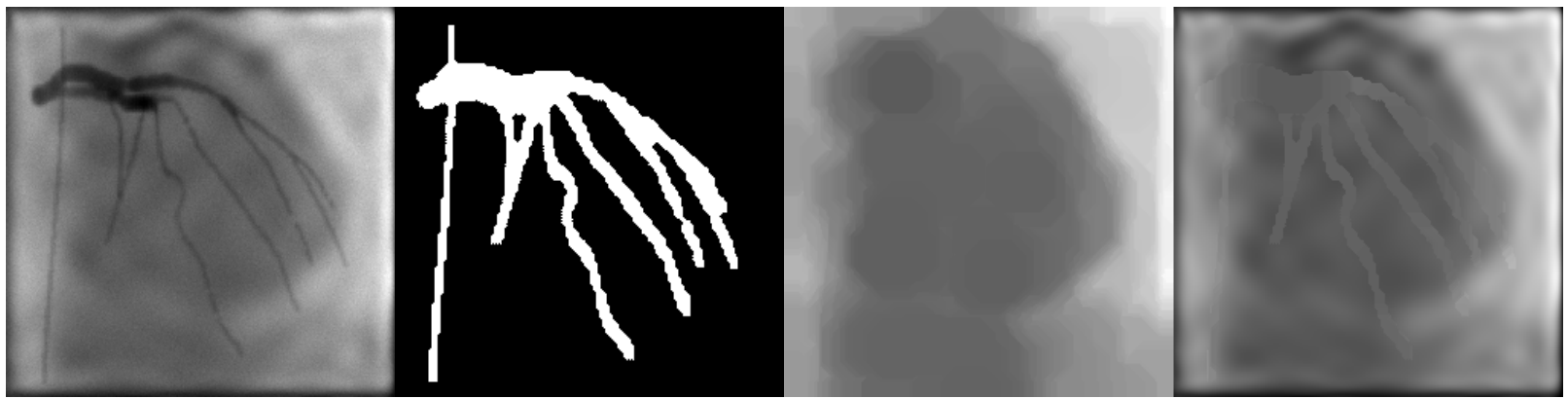

Figure 4. Figures from left to right are 1 ) the projection image at angle $-5.4^{\circ} ; 2$ ) the corresponding binary vessel mask where the white color indicates the valid vessel regions. 3) the projection image after a morphological closure filtering with a disk type structure element of radius size 10 pixels; 4) the resulting virtual background image.

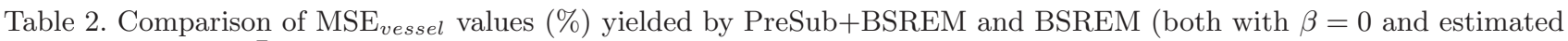
background image $\overline{\hat{\boldsymbol{Y}}}_{\mathrm{b}}$ ) over iterations.

\begin{tabular}{c|c|c|c|c}
\hline \#Itr. & 5 & 30 & 70 & 100 \\
\hline PreSub+BSREM & 39.9 & 27.2 & 28.8 & 29.7 \\
BSREM & 57.4 & 26.1 & 27.7 & 27.2 \\
\hline
\end{tabular}




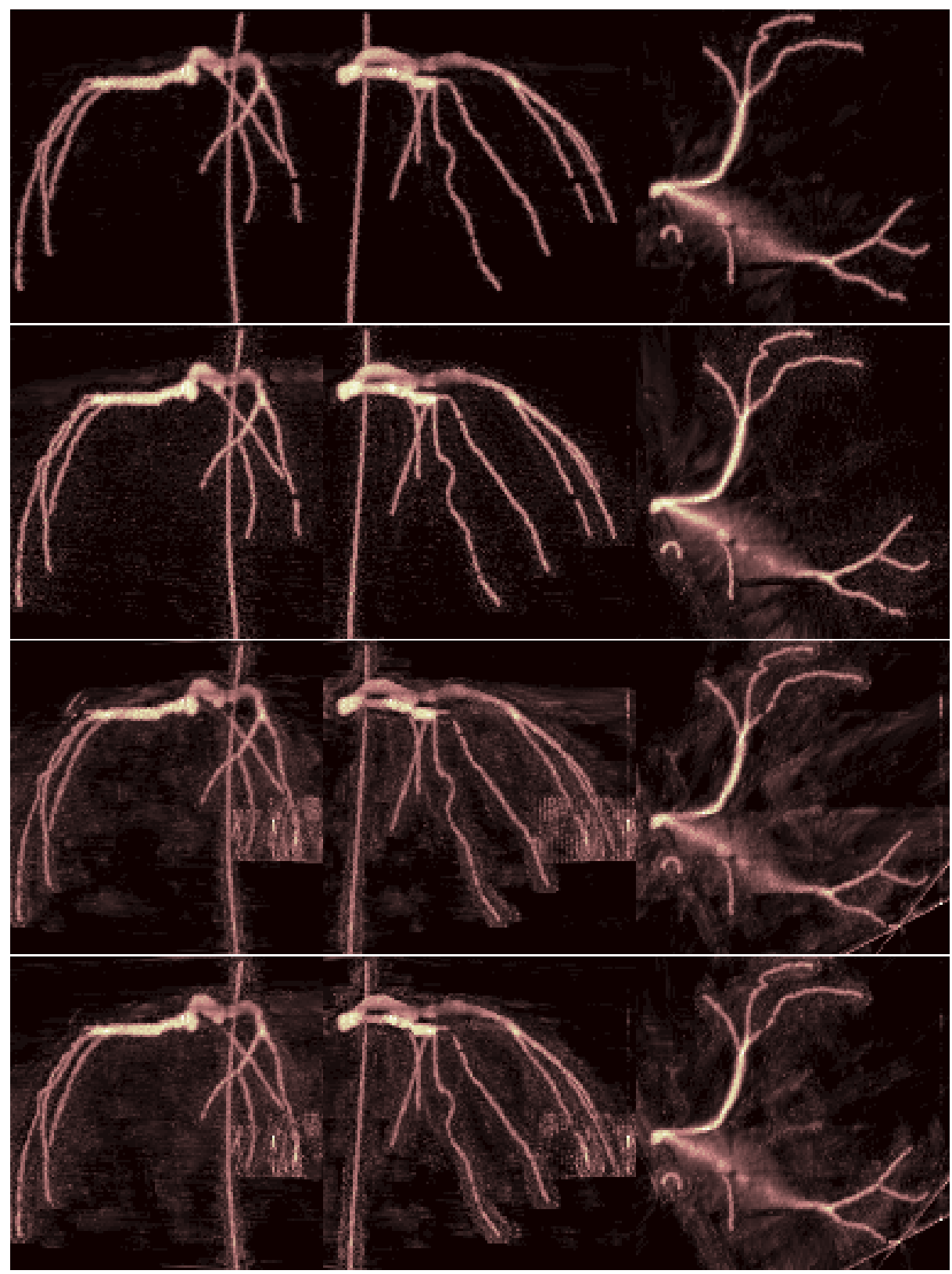

Figure 5. From top to bottom: maximum intensity projection images of reconstructions by using: BSREM (with $\hat{\boldsymbol{Y}}_{\mathrm{b}}$ ), PreSub+BSREM (with $\hat{\boldsymbol{Y}}_{\mathrm{b}}$ ), BSREM (with $\overline{\hat{\boldsymbol{Y}}}_{\mathrm{b}}$ ) and PreSub+BSREM (with $\overline{\hat{\boldsymbol{Y}}}_{\mathrm{b}}$ ), respectively. ( $\beta=0$ for all algorithms)

\subsection{Choose image prior}

This section evaluates the impact of the prior model on the $3 \mathrm{D}$ vessel reconstruction. From our former analysis, here the main task of prior is to reduce the background noise while preserving main vascular structures undestroyed. The comparison has been conducted between a heavy-tailed model and a non-heavy-tailed model. For the latter, we used the Gaussian quadratic prior model (where $\phi(t)=|t|^{2}$ ). The choice of parameter $\beta$ is essential due to its smoothing effects. On the other hand, for a specific prior, the choice of $\beta$ also can be different. Thus, for a fair comparison, we selected these model-based parameter with the aim to minimize the MSE $\mathrm{vessel}$ error. More precisely, we chose several $\beta$ values and recorded the corresponding the minima $\mathrm{MSE}_{\text {vessel }}$ value of reconstruction during iterations. We can trace out the relationship on the $\beta$ and $\mathrm{MSE}_{\text {vessel }}$ plane. An interpolation between discrete samples is then made to predict a global optimal $\beta$. Fig. 6 shows two curves related to 


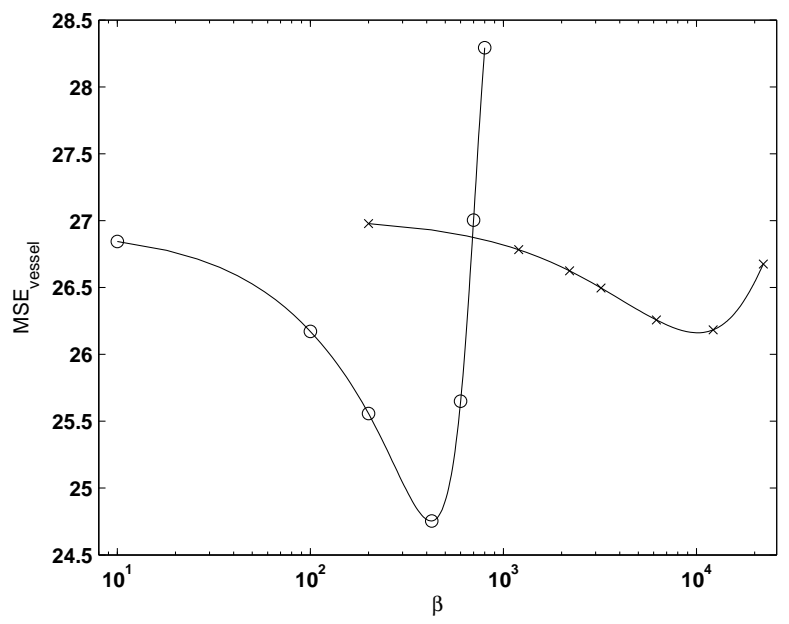

Figure 6. A comparison of the minimum $\mathrm{MSE}_{\text {vessel }}$ changes as a function of parameter $\beta$ yielded by BSREM using different priors: quadratic prior $\left(-x_{-}\right)$and total variation like prior $(-\circ-)$. These two curves are generated by interpolation where discrete samples are indicated by symbols, i.e., circles and crosses. The optimal $\beta$ values are approximately $\beta=10^{4.0}$ for the quadratic prior, and $\beta=10^{2.62}$ for TV-like prior.

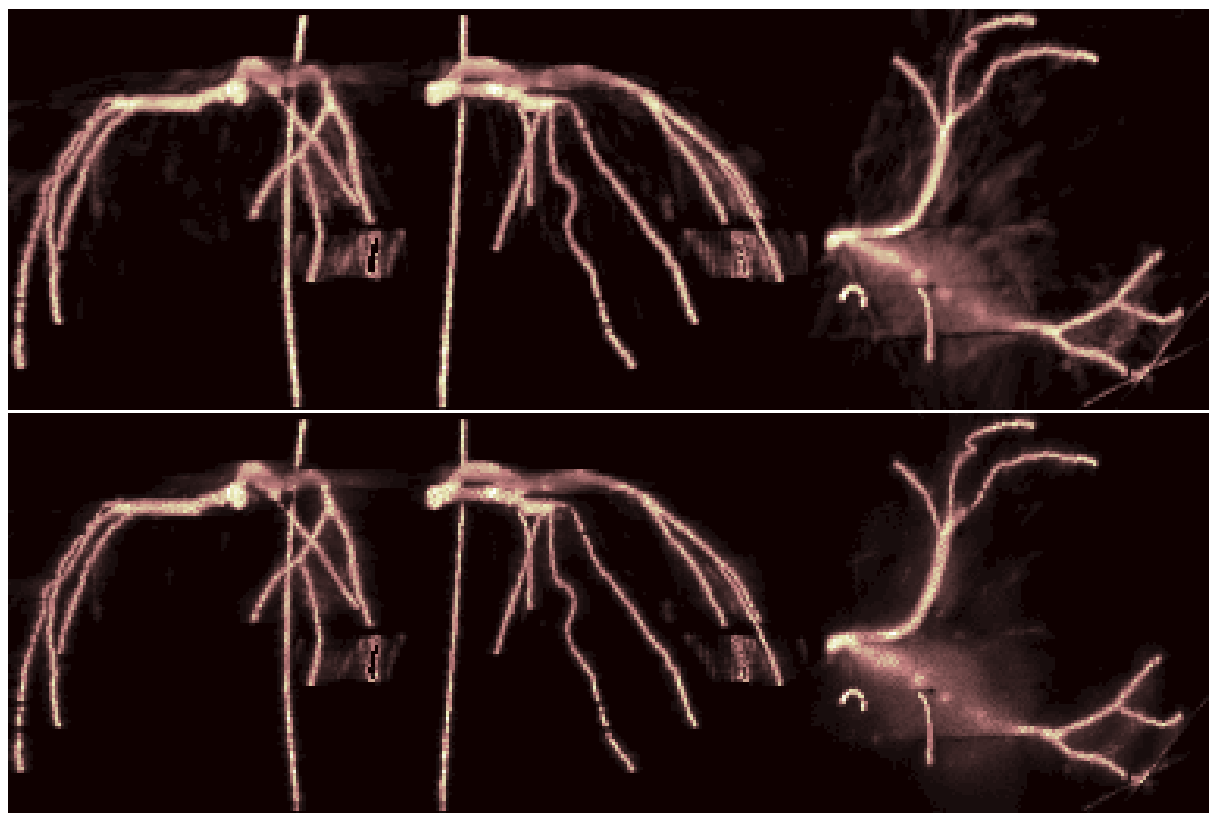

Figure 7. A comparison of MIP of image reconstruction from BSREM using: the quadratic prior with $\beta=10^{4.0}$ (the first row) and the TV-like prior with $\beta=10^{2.62}$ (the second row).

different priors. In our experiments, the optimal $\beta$ for the quadratic prior is close to $10^{4.0}$ while for a TV-like prior it is about $10^{2.62}$. Note that the global minimum $\mathrm{MSE}_{\text {vessel }}$ yielded by a TV-like prior is smaller than a quadratic prior, which points out the advantage of TV-like prior over a quadratic one in terms of reconstruction performance.

The results (MIP images) are displayed in Fig. 7. It is clear that the introduction of prior leads to a successful suppression of background artifacts (Note that here we used the virtual background image instead of the true one). From a visual point of view, we also see that the sparse prior, as well as the TV-like prior, can provide even better result (a clear comparison from the top-to-down MIP images). Fig. 8 shows further visual comparisons by using $3 \mathrm{D}$ vessel surface rendering. All surfaces are rendered by using the same isosuface values extracted from 


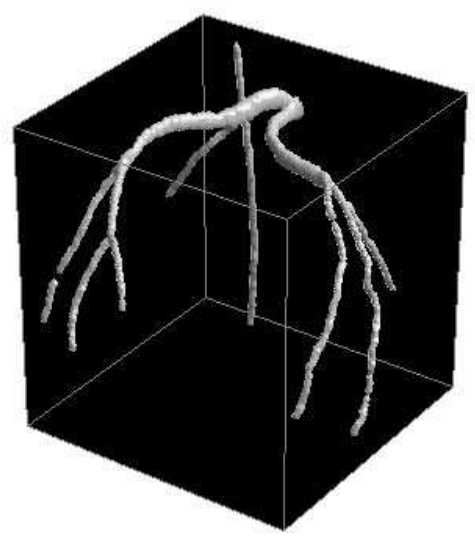

(a)

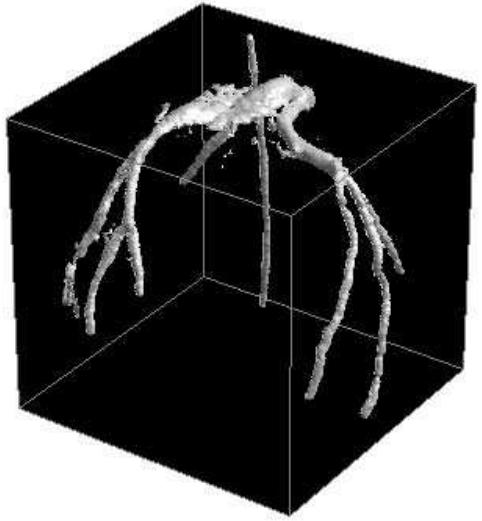

(b)

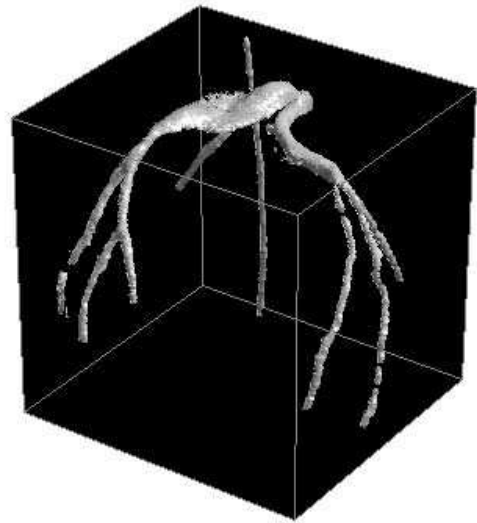

(c)

Figure 8. 3D vascular surface rendering using true vessel data (a), and data reconstructed by BSREM with the quadratic prior (b), and the TV-like prior (c).

various reconstructed volumes. Again a better result is obtained by the proposed TV-like prior. However, it is worth to note that both priors still fail to reconstruct some vessel parts marked with circles in Fig. 8. Thus, the introduction of prior models may not fulfil the requirements due to the incomplete rotational X-ray scanning.

\subsection{The effect of data inconsistency}

Till now, the used projection images were motion free data (i.e., the same cardiac phase). In real case, the key assumption relies on the fact that the coronary network is observed exactly in the same position for different views: The reproducibility of the heart cycle and the temporal sampling may introduce some spatial shift. In other words, some inconsistency may exist. Two main motions were examined: rotation and translation. We simulated this data mismatch to explore how it affects the reconstruction. The heart rotation has been simulated by using a random bias, denoted by $\epsilon_{\text {angle }}$, added to the projection angle. For translation, since the cardiac motion is mainly dominated by a up-down movement, we used a random up-down displacement $\epsilon_{\text {displacement }}$ to change the real vessel position when generating each projection image. We only studied the BSREM algorithm with the TV-like prior, and the virtual background images were estimated according to the same method described before. No pre-subtraction was used.

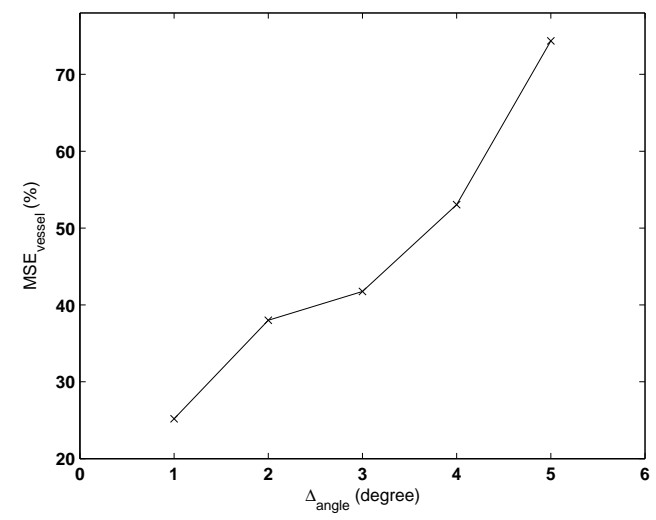

(a)

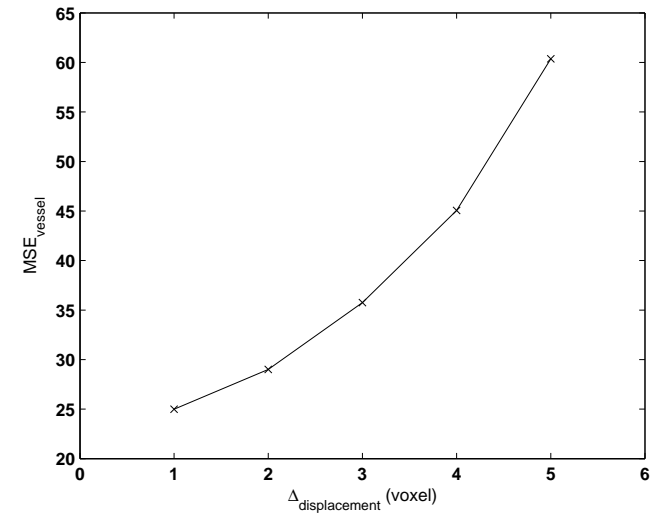

(b)

Figure 9. (a) The changes of $\mathrm{MSE}_{\text {vessel }}$ with $\Delta_{\text {angle }}$ when using the proposed BSREM algorithm coupled with a TV-

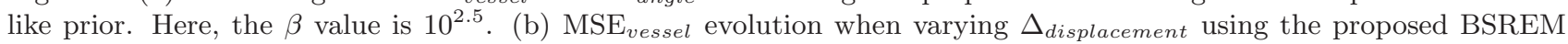
algorithm coupled with a TV-like prior. Here, the $\beta$ value is $10^{2.5}$. 
Fig. 9(a) plots about the changes of $\mathrm{MSE}_{\text {vessel }}$ against the maximum magnitude of $\epsilon_{\text {angle }}$, i.e., $\Delta_{\text {angle }}$. Note that $\epsilon_{\text {angle }}$ is the uniform random number within the range $\left[-\Delta_{\text {angle }},+\Delta_{\text {angle }}\right]$. We see clearly that the mean square error increases with error in angle. For an amount of $5^{\circ}$ bias in angle, it results in almost three times larger error in reconstruction. Fig. 9(b) shows the relationship between $\mathrm{MSE}_{\text {vessel }}$ and the object displacement error $\epsilon_{\text {displacement }}$ (note also that $\epsilon_{\text {displacement }}$ is the uniform random number within the range $\left.\left[-\Delta_{\text {displacement }},+\Delta_{\text {displacement }}\right]\right)$. The same behavior can be observed. They both show that if the assumption made does not hold, a significant degeneration in the quality of reconstruction can be anticipated.

\section{DISCUSSION AND CONCLUSION}

We have described in this paper a method for the 3D reconstruction of coronary networks from sparse projections acquired through a rotational system within a limited angle rotation. This approach, applied in a first step in static conditions by assuming an error-free ECG gating, is based on a statistical modelling, blob basis functions (Kaiser-Bessel) and makes use of a block iterative algorithm (BSREM, block sequential regularized expectationmaximisation). Simulations have been performed by means of a realistic phantom representing an arterial tree extracted from a MDCT dataset in order to have a ground truth. The performance of the method has been assessed by using global statistics (mean square values over the reconstructed volume or over the vessel spatial support). The reconstructions that have been reported show some promising features. They also allow quantifying the influence of the background and the sensitivity to imprecise synchronization resulting in object misregistration (simulated by small translation and rotation errors).

It must be said that the reconstruction of coronary network remains a difficult problem in X-ray imaging (including the MDCT) for several reasons among which: the small size of the objects (few millimeters), the many structures contributing to the background, the patient movements, the ballistic inaccuracies and the time-varying heart cycle. Although this work-in-progress has been limited to a cone beam static situation, it opens a path to deal with dynamic reconstruction. In contrast to approaches that call first for 3D motion field estimation with feature matching, the solution that we are currently developing is based on a fully homogeneous tomographic frame. The availability of 3D dataset (single volume or time indexed multiple volumes) as shown here should serve as an initialization step for further refinements.

\section{ACKNOWLEDGMENTS}

This work was supported by an INSERM postdoctoral grand, a PhD grant from the University of Rennes 1 , and the scholarship of "co-tutelle en thèse" by French government.

\section{REFERENCES}

1. M. Garreau, J. L. Coatrieux, R. Collorec, and C. Chardenon, "A knowldege-based approach for 3D reconstruction and labeling of vascular network from biplance angiographic projections," IEEE Trans. Med. Imaging, 10, pp. 122-131, 1991.

2. C. Venaille, D. Mishler, and J. L. Coatrieux, "Un algorithme peu contraint d'appariement de primitives courbes par stéréovision trnoculaire," Rev. Technique-Thomson-CSF, 24, pp. 1071-1099, 1992.

3. S. Ruan, A. Bruno, and J. L. Coatrieux, "Three dimensional motion and reconstruction of coronary arteries from biplane cineangiography," Image and Vision Computing, 12, pp. 683-689, 1994.

4. J. L. Coatrieux, M. Garreau, R. Collorec, and C. Roux, "Computer vision approaches for the threedimensional reconstruction of coronary arteries: review and prospects," Crit. Rev. Biomed. Eng., 22, pp. $1-38,1994$.

5. S. Bonnet, A. Koenig, S. Roux, P. Hugonnard, R. Guillemaud, and P. Grangeat, "Dynamic X-ray computed tomography," Proc. IEEE, 91, pp. 1574-1587, 2003.

6. C. Blondel, G. Malandain, R. Vaillant, and N. Ayache, "Reconstruction of coronary arteries from a single rotational X-ray projection sequence," IEEE Trans. Med. Imaging, 25, pp. 653-663, 2006.

7. U. Jandt, D. Schäfer, V. Rasche, and M. Grass, "Automatic generation of 3D coronary artery centerlines using rotational X-ray angiography," in Proc. SPIE Medical Imaging, 6510, 2007. 
8. E. Hansis, D. Schäfer, G. Michael, and O. Dössel, "An iterative method for the reconstruction of the coronary arteries from rotational X-ray angiography," in Proc. SPIE Medical Imaging, 6510, 2007.

9. B. Movassaghi, M. Grass, D. Schäfer, V. Rasche, O. Wink, G. Schoonenberg, J. Y. Chen, J. A. Garcia, B. M. Groves, J. C. Messenger, and J. D., Carroll, "4D coronary reconstruction based on retrospectively gated rotational angiography: first in-human results," in Proc. SPIE Medical Imaging, 6509, 2007.

10. A. De Pierro and M. E. B. Yamagishi, "Fast scaled gradient decomposition methods for maximum likelihood transmission tomography," in Proceedings of the 25th Annual International Conference of the IEEE Engineering in Medicine and Biology Society, 1, pp. 829-832, 2003.

11. R. Lewitt, "Multidimensional digital image representation using generalized Kaiser-Bessel window functions," J. Opt. Soc. Am. A., 7, pp. 1834-1846, 1990.

12. S. Matej and R. Lewitt, "Practical considerations for 3D image reconstruction using spherically symmetric volume elements," IEEE Trans. Med. Imaging, 15, 68-78.

13. M. E. Daube-Witherspoon, S. Matej, and J. S. Karp, "Assessment of image quality with a fast fully 3D reconstruction algorithm," in Proceedings of Nuclear Science Symposium Conference Record, 4, pp. 2238$2242,2001$.

14. W. L. Wang, W. Hawkins, and D. Gagnon, "3D RBI-EM reconstruction with spherically-symmetric basis function for SPECT rotating slat collimator," Phys. Med. Biol, 49, pp. 2273-2292, 2004.

15. S. Muraki, "Volumetric shape desciption of range data using 'Blobby Model'," Computer Graphics, 25, pp. 227-235, 1991.

16. G. Yang, A. Bousse, C. Toumoulin, and H. Shu, "A multiscale tracking algorithm for the coronary extraction in MSCT angiography," In Proceedings of Engineering in Medicine and Biology Society, 2006. EMBS '06. 28th Annual International Conference of the IEEE, pp. 3066-3069, 2006.

17. J. Browne and A. R. De Pierro, "A row-action alternative to the EM algorithm for maximizing likelihood in emission tomography," IEEE Trans. Med. Imaging, 15, pp. 687-699, 1996.

18. R. Gordon, R. Bender and G.T. Herman, "Algebraic reconstruction techniques (ART) for three-dimensional electron microscopy and x-ray photography," Journal of Theoretical Biology, 29, pp. 471-482, 1970. 